\title{
Commentary: High Expression of Cancer-IgG Is Associated With Poor Prognosis and Radioresistance via PI3K/AKT/DNA-PKcs Pathway Regulation in Lung Adenocarcinoma
}

\author{
Liu Yang ${ }^{1 \dagger}$, Pingan $\mathrm{Lu}^{2 \dagger}$ and Song $\mathrm{Qu}{ }^{1,3 *}$ \\ ${ }^{1}$ Department of Radiation Oncology, Guangxi Medical University Cancer Hospital, Nanning, China, ${ }^{2}$ Amsterdam UMC, \\ Faculty of Medicine, University of Amsterdam, Amsterdam, Netherlands, ${ }^{3}$ Key Laboratory of High-Incidence Tumor \\ Prevention \& Treatment (Guangxi Medical University), Ministry of Education, Nanning, China
}

Keywords: neoplasms, radioresistance, DNA-PK, cancer-derived IgG, normal tissue toxicity

\section{OPEN ACCESS}

Edited by:

John Varlotto,

Marshall University, United States

Reviewed by:

Valentyn Oksenych,

University of Oslo, Norway

*Correspondence:

Song Qu

13607887386@163.com

${ }^{\dagger}$ These authors have contributed equally to this work and

share first authorship

Specialty section:

This article was submitted to

Radiation Oncology,

a section of the journal

Frontiers in Oncology

Received: 14 July 2021 Accepted: 20 September 2021 Published: 07 October 2021

Citation:

Yang L, Lu P and Qu S (2021) Commentary: High Expression of Cancer-lgG Is Associated With Poor

Prognosis and Radioresistance via

PIJK/AKT/DNA-PKcs Pathway Regulation in Lung Adenocarcinoma.

Front. Oncol. 11:741089.

doi: 10.3389/fonc.2021.741089

\section{A Commentary on}

High Expression of Cancer-IgG Is Associated With Poor Prognosis and Radioresistance via PI3K/AKT/DNA-PKcs Pathway Regulation in Lung Adenocarcinoma

By Yang X, Wang G, You J, Gu R, Xu X, Xu C, Wang H, Zhao R, Qiu X and Zhu G (2021). Front. Oncol. 11:675397. doi: 10.3389/fonc.2021.675397

We have read with great interest the article recently published by Yang and colleagues (1), in which surgical tissue specimens from 56 lung adenocarcinoma (LUAD) patients as well as LUAD cell lines H292, A549, H1299 and PC9 were employed to excavate the functional link of cancer-derived IgG with acquired radioresistance. The high expression of cancer-IgG in LUAD tissues and cell lines and its correlation with inferior clinical outcomes (1) concur with previous findings in clear cell renal cell carcinoma (2), ovarian cancer (3), colorectal cancer (4) and salivary adenoid cystic carcinoma (5), which reinforced the concept that cancer-IgG plays an indispensable role in malignant transformation and tumor progression. Furthermore, this study is arguably the first study demonstrating that cancer-IgG overexpression could substantially promote the post-irradiation phosphorylation of DNA-dependent protein kinase catalytic subunit (DNA-PKcs). This valuable finding is in our opinion worth of a further emphasis.

DNA-PK is a key effector of the non-homologous end-joining (NHEJ) pathway, which is the predominant DNA damage repair machinery used by tumor cells to combat ionizing radiation (IR) induced DNA double strand breaks. Over the past decades, myriad research groups and pharmaceutical companies have made great efforts to develop novel pharmaceutical compounds that could potently and specifically inhibit DNA-PK to potentiate IR-induced cytotoxicity in radioresistant neoplasms. However, the cardinal principle of radiation oncology - maximizing dose to tumor while minimizing damage to surrounding normal tissues - has not received sufficient attention in this treatment strategy. As normal cells also need the expression and activation of DNAPK to survive IR-insult, systemic administration of DNA-PK inhibitors (DNA-PKIs) in combination with radiotherapy may exacerbate normal tissue toxicity as much as tumor toxicity. For example, the 
development of DNA-PKIs wortmannin and LY294002 has been discontinued at preclinical phase due to severe systemic toxicity in mouse models (6). The same apprehension was further raised by data released from an ongoing phase I trial (NCT02516813) testing the tolerability of DNA-PKI M381 in combination with radiotherapy, which reported several forms of normal tissue toxicity including dysphagia, prolonged mucosal inflammation/ stomatitis and radiation skin injury (7). Unsurprisingly, these normal tissue reactions were not observed in another phase I trial (NCT02316197) evaluating the tolerability of M381 as monotherapy (8).

Currently, attempts towards finding novel treatment strategies that selectively target the NHEJ-mediated DSB repair in tumors are still ongoing, exemplified by the DNA-PKI prodrug that could be selectively activated in the hypoxic tumor microenvironment (9). In this regard, we would like to voice the great significance of the findings by Yang and colleagues (1). Since cancer-IgG is specifically secreted by cancer cells and is capable of promoting the phosphorylation

\section{REFERENCES}

1. Yang X, Wang G, You J, Gu R, Xu X, Xu C, et al. High Expression of CancerIgG Is Associated With Poor Prognosis and Radioresistance via PI3K/AKT/ DNA-PKcs Pathway Regulation in Lung Adenocarcinoma. Front Oncol (2021) 11:675397. doi: 10.3389/fonc.2021.675397

2. Sheng Z, Liu Y, Qin C, Liu Z, Yuan Y, Hu F, et al. IgG Is Involved in the Migration and Invasion of Clear Cell Renal Cell Carcinoma. J Clin Pathol (2016) 69(6):497-504. doi: 10.1136/jclinpath-2015-202881

3. Ji F, Chang X, Liu C, Meng L, Qu L, Wu J, et al. Prognostic Value and Characterization of the Ovarian Cancer-Specific Antigen CA166-9. Int J Oncol (2015) 47(4):1405-15. doi: 10.3892/ijo.2015.3115

4. Jiang H, Kang B, Huang X, Yan Y, Wang S, Ye Y, et al. Cancer IgG, a Potential Prognostic Marker, Promotes Colorectal Cancer Progression. Chin J Cancer Res = Chung-kuo yen cheng yen chiu (2019) 31(3):499-510. doi: 10.21147/ j.issn.1000-9604.2019.03.12

5. Peng J, Wang HC, Liu Y, Jiang JH, Lv WQ, Yang Y, et al. Involvement of Non-B Cell-Derived Immunoglobulin G in the Metastasis and Prognosis of Salivary Adenoid Cystic Carcinoma. Oncol Lett (2017) 14(4):4491-8. doi: 10.3892/ ol.2017.6782

6. Collis SJ, DeWeese TL, Jeggo PA, Parker AR. The Life and Death of DNA-Pk. Oncogene (2005) 24(6):949-61. doi: 10.1038/sj.onc.1208332

7. Mau-Sorensen M, van Bussel M, Kuipers M, Nielsen DL, Verheul HM, Aftimos $\mathrm{P}$, et al. Safety, Clinical Activity and Pharmacological Biomarker Evaluation of the DNA-Dependent Protein Kinase (DNA-PK) Inhibitor M3814: Results From Two Phase I Trials. Ann Oncol (2018) 29:viii654. doi: 10.1093/annonc/ mdy303.015 of DNA-PKcs, targeted pharmacological inhibition of cancerIgG holds the promise to significantly circumvent the problematic normal tissue toxicities of traditional DNA-PKIs while providing similar radiosensitizing effects. Additionally, as cancer-IgG expression was not only observed in LUAD (2-5), multifaceted therapeutic benefits of anti-cancer-IgG agents could also be expected for radioresistant patients with other malignant diseases, as well as for patients suffering from intrinsic or acquired resistance to DNA-damaging chemotherapeutics.

\section{AUTHOR CONTRIBUTIONS}

LY and PL performed literature research and drafted the manuscript. SQ supervised the study and reviewed the manuscript. All authors contributed to the article and approved the submitted version. LY and PL contributed equally as co-first authors.

8. van Bussel MTJ, Awada A, de Jonge MJA, Mau-Sørensen M, Nielsen D, Schöffski P, et al. A First-in-Man Phase 1 Study of the DNA-Dependent Protein Kinase Inhibitor Peposertib (Formerly M3814) in Patients With Advanced Solid Tumours. Br J Cancer (2021) 124(4):728-35. doi: 10.1038/ s41416-020-01151-6

9. Wong WW, Jackson RK, Liew LP, Dickson BD, Cheng GJ, Lipert B, et al. Hypoxia-Selective Radiosensitisation by SN38023, A Bioreductive Prodrug of DNA-Dependent Protein Kinase Inhibitor IC87361. Biochem Pharmacol (2019) 169:113641. doi: 10.1016/j.bcp.2019.113641

Conflict of Interest: The authors declare that the research was conducted in the absence of any commercial or financial relationships that could be construed as a potential conflict of interest.

Publisher's Note: All claims expressed in this article are solely those of the authors and do not necessarily represent those of their affiliated organizations, or those of the publisher, the editors and the reviewers. Any product that may be evaluated in this article, or claim that may be made by its manufacturer, is not guaranteed or endorsed by the publisher.

Copyright (c) 2021 Yang, Lu and Qu. This is an open-access article distributed under the terms of the Creative Commons Attribution License (CC BY). The use, distribution or reproduction in other forums is permitted, provided the original author(s) and the copyright owner(s) are credited and that the original publication in this journal is cited, in accordance with accepted academic practice. No use, distribution or reproduction is permitted which does not comply with these terms. 